\title{
Osteoporosis care amidst the prolonged pandemic
}

\author{
R. R. Narla ${ }^{1,2} \cdot$ R. A. Adler ${ }^{3,4}$
}

Received: 3 January 2021 / Accepted: 20 February 2021 / Published online: 28 February 2021

(c) This is a U.S. government work and not under copyright protection in the U.S.; foreign copyright protection may apply 2021

\begin{abstract}
As SARS-CoV-2 stunned and overtook everyone's lives, multiple daily briefings, protocols, policies and incident command committees were mobilized to provide frontline staff with the tools, supplies and infrastructure needed to address the COVID-19 pandemic. Medical resources were immediately shifted. In light of the necessity for self-isolation, telemedicine was expanded, although there has been concern than non-pandemic disorders were being ignored. Ambulatory care services such as bone densitometry and osteoporosis centered clinics came to a near halt. Progress with fracture prevention has been challenged. Despite the prolonged pandemic and the consequent sense of exhaustion, we must re-engage with chronic bone health concerns and fracture prevention. Creating triaging systems for bone mineral testing and in person visits, treating individuals designated as high risk of fracture using fracture risk assessment tools such as FRAX, maintaining telemedicine, leveraging other bone health care team members to monitor and care for osteoporotic patients, and re-engaging our primary care colleagues will remain paramount but challenging. The pandemic persists. Thus, we will summarize what we have learned about COVID-19 and bone health and provide a framework for osteoporosis diagnosis, treatment, and follow-up with the extended COVID-19 pandemic. The goal is to preserve bone health, with focused interventions to sustain osteoporosis screening and treatment initiation/maintenance rates.
\end{abstract}

Keywords Osteoporosis · COVID-19 · Telemedicine · Bone Mineral Density · Fracture Prevention

\section{Introduction}

All across the world COVID-19 rages, leading to crippling acute losses and unknown long term consequences. Many countries have reverted to earlier restrictions, re-entering near or full lockdown measures to limit the rapid spread of this virus. Even with the start of vaccination programs,

R. A. Adler

Robert.Adler@va.gov

1 Division of Metabolism, Endocrinology and Nutrition, Department of Medicine, School of Medicine, University of Washington, Seattle, WA, USA

2 Division of Endocrinology, Metabolism and Nutrition, Veterans Affairs Puget Sound Health Care System, Seattle, WA, USA

3 Endocrinology and Metabolism Section (111P), McGuire Veterans Affairs Medical Center, Central Virginia Veterans Affairs Health Care System, 1201 Broad Rock Boulevard, Richmond, VA 23249, USA

4 Division of Endocrinology, Metabolism, and Diabetes Mellitus, Virginia Commonwealth University, Richmond, VA, USA other public health measures will continue to be necessary for months. Health care systems are challenged to meet the demands of the acute care needs for COVID-19 patients, vaccinate millions, and preserve medical resources. Outpatient facilities are again restricting services. Finally, as more people remain at home or work remotely, the reluctance to seek care for non-COVID-19-related concerns, including for bone health, continues to grow.

Recent advancements in osteoporosis management, including more treatment options such as anabolic agents, have led to better opportunities to increase bone mineral density and reduce fracture risk. In spite of this, millions of people worldwide remain at risk for osteoporotic fractures. In the United States (US), an estimated 2 million people sustain fragility fractures each year [1]. A report that included six of the largest European countries (France, Germany, Italy, Spain, United Kingdom and Sweden) estimated the total number of fragility fractures to increase from 2.7 million in 2017 to an astonishing 3.3 million in 2030 [2]. With these alarming numbers, the economic burden of osteoporosisrelated fracture is marked, costing close to $\$ 17.9$ billion and $£ 4$ billion per annum in the USA and UK, respectively. [3] 
Despite devastating consequences of osteoporotic fractures on quality and quantity of life, mobility, and independence of living, there remain major barriers and limitations to case detection, screening and treatment options, especially for the aging population. There are challenges to both primary and secondary fracture prevention.

Osteoporosis does not take a break while COVID-19 rages on. Healthcare providers should resume primary care services and preventive medicine as soon as it is deemed safe, appropriate and practical. Metabolic bone experts should identify local barriers to screening and case detection of osteoporosis whilst mitigating system level issues and supporting primary care. Fracture prevention is particularly challenging because people are weary and frustrated by the pandemic. In addition, they are fearful about returning to healthcare facilities for necessary metabolic laboratory testing or required in person visits, such as bone mineral density measurements. We must apply what has been learned so far from the pandemic to the detection and management of osteoporosis, as the infections, hospitalizations, and deaths continue.

\section{What impact on osteoporosis care have we witnessed during pandemic?}

As an illustration of the impact of the pandemic, one orthopedic hospital in Italy noted an almost $50 \%$ reduction in bone densitometry testing, compared to similar months in 2019, with a complete halt of testing in April 2020 [4]. As systems open up, anticipated wait times for Dual-energy $\mathrm{X}$-ray absorptiometry (DXA) testing will likely increase markedly.

It is possible to assess fracture risk in some patients via telemedicine or telephone modalities by calculating 10-year fracture risk using (FRAX ${ }^{\circledR}$, www.shef.ac.uk/FRAX) without DXA measurements, available in 66 countries. There are alternate fracture risk calculators. Most bone health organizations agree that if the fracture risk threshold is met using this tool (which essentially uses body mass index as a surrogate for bone density), clinicians should consider empiric treatment [5]. Despite this, McCloskey and colleagues recently reported a significant reduction in access to the FRAX website, since COVID-19 crisis began. The reduction averaged 58\% with less drop in Asian countries. In North America, decreases in usage by April 2020 were greater for the USA than for Canada ( $-60.9 \%$ and $-44.9 \%$, respectively) while in Europe, some countries such as Spain, Portugal, Greece, Malta, Georgia and Slovenia had decreased use of the FRAX website by more than 75\% [6].

In a large secondary care hospital in the UK, the average number of outpatients (aged $\geq 50$ years) attending a single Fracture Clinic for a new suspected non-hip fragility fracture was lower during lockdown in 2020. However, during the same time period, no change was observed in the mean number of new inpatient admissions for acute hip fracture [7]. Initial data suggested a different distribution of fractures with more occurrence of shoulder and elbow injury due to low-energy trauma or falls in residences [8]. Other studies also found that the overall incidence of fragility fractures was unaffected by the pandemic $[9,10]$.

\section{What bone health comorbidities should we be vigilant about associated with COVID-19?}

\section{Systemic corticosteroids}

Dexamethasone and other systemic corticosteroids have been used to dampen the cytokine storm in acutely ill COVID-19 patients. While often used short-term, glucocorticoids' risks and benefits must be addressed, including dose and duration given the risks of bone loss and avascular necrosis, as suggested by Zhang et al. [11].

\section{COVID-19 and vertebral fractures}

A potential outcome predictor of COVID-19 infection may be the presence of vertebral fractures (VF). In a retrospective cohort study of Italian patients aged $\geq 18$ years with the confirmed diagnosis of COVID-19 at a tertiary care hospital, 114 had lateral chest X-rays. Thoracic VFs were identified in 41 patients, 19 of whom had multiple VFs. Patients with VFs were older and had comorbidities such as hypertension and coronary artery disease $(P<0.001, P=0.007, P=0.034$, respectively). More patients with VFs required hospitalization and nearly half of the VF subjects needed non-invasive mechanical ventilation in the hospital compared with $27 \%$ of the COVID-19 patients without VFs $(P=0.02)$. Lastly, the mortality was somewhat higher in the VF patients but not statistically significant [12]. VFs often remain undiagnosed, negatively affecting physical functioning, presumably by a restrictive lung defect. While the patients in this study had comorbidities beyond VF, the question can be asked: would the diagnosis and treatment of osteoporosis prior to infection have affected the course of the COVID-19 illness?

\section{Racial disparities and bone health}

Most studies that have evaluated outcomes after fragility fractures have been conducted in Caucasian populations, but a few show racial disparities in bone health outcomes. $[13,14]$ Caucasian women have higher rates of hip fracture, but black women have higher morbidity and mortality [15, 16]. A recent observational cohort study using US Medicare data from 2010 to 2015 by Wright et al. [14] found that black women had higher 1-year mortality (19.6\% vs. $15.4 \% ; P<0.001)$ and higher 1 -year incidence of debility 
following femur fractures (6.6\% vs. 3.9\%) compared with white women. Debility was defined as new placement in a long term nursing facility, site of many COVID-19 outbreaks. In the US, black and Hispanic populations appear to be at higher mortality risk from COVID-19.

\section{Hyperparathyroidism and surgery during the pandemic}

From the evaluation of secondary osteoporosis, patients may be diagnosed with concomitant primary hyperparathyroidism (PHPT) and meet criteria for surgery [17]. During the pandemic patients with PHPT will be conservatively treated and monitored, unless the patient becomes severely symptomatic with significantly elevated serum calcium levels. If access to surgery is not possible, cinacalcet could be considered with bisphosphonates used to preserve bone. Many surgery centers have created algorithms to prioritize surgery for HPTH $[18,19]$.

\section{COVID-19 and Vitamin D}

Several studies have addressed the potential association of low serum 25-hydroxyvitamin D [25(OH)D] levels and risk of COVID-19 infection and/or seriousness of infection [20-22]. Proposed mechanisms have been outlined recently by Bilezikian et al. including vitamin $\mathrm{D}$ modulating the innate and acquired immune system, production of antimicrobial peptides cathelicidin and human $\beta$-defensin- 2 as well as potential vitamin $\mathrm{D}$ inhibition of pro-inflammatory cytokine production observed in SARS-CoV-2 infection [23]. However, as noted by others, low 25(OH)D levels are already observed in patients with other medical conditions such as diabetes mellitus, chronic pulmonary conditions; and these patients are known to be at higher risk for COVID19. Nonetheless, Hernandez et al. noted that Vitamin D deficiency was found in $82.2 \%$ of COVID-19 cases versus $47.2 \%$ of population-based controls $(P<0.0001)$, even after adjusting for age, smoking, hypertension, diabetes mellitus, history of cardiovascular events, immunosuppression, body mass index, serum corrected calcium, and glomerular filtration rate [21]. Serum $25(\mathrm{OH}) \mathrm{D}$ has been considered as a negative acute-phase reactant, and values have been reported to be lower during acute inflammatory diseases [24]. While others have also highlighted the importance of vitamin D's potential role in muscle health and extra-skeletal benefits in the advanced aging population, the studies are ongoing to further elucidate these properties [25]. Thus, a consistent message to patients with known osteoporosis and perhaps to the population in general is that dietary recommendations for bone health may have other salutary consequences. It is thus important to review calcium and protein intake, and vitamin $\mathrm{D}$ doses per bone health guidelines.

\section{Differences in COVID-19 in men vs. women: do sex steroids play a role?}

Some initial reports regarding COVID-19 found gender differences with men having more serious cases than women [26]. In a retrospective study from Spain, men's vitamin D $25 \mathrm{OH}$ levels were lower than women's [21]. In an Italian study, VFs were detected in 35\% of COVID-19 male patients and in $37 \%$ of female patients, a finding which seemed higher than estimated incidence of vertebral fracture in many US or European populations [12]. While life-style factors and other etiologies could be important, one speculation has been that estrogen plays a protective effect on the immune system [27]. Because of this hypothesis, there are some initial studies using raloxifene, a selective estrogen receptor modulator, as an inhibitor of IL-6, especially in severe COVID-19 cases [28]. More studies are necessary to determine if the apparent gender difference is mediated via sex steroids.

\section{Initiating osteoporosis treatment options during the pandemic}

In the initial weeks of the pandemic, there was some hesitation to initiate new pharmacotherapy for osteoporosis, perhaps due to the expectation that the pandemic would subside. With prolongation of the pandemic despite the promise of the multiple vaccines, social distancing, mask wearing, and minimization of travel will continue for several more months. Clinicians need to maintain clear and informative shared decision making discussions with patients about the benefits and risks of their osteoporosis treatment. Osteoporosis treatment should not be postponed in most cases. Initiating oral bisphosphonates using telemedicine is relatively safe and effective [29]. Initiating intravenous bisphosphonates, denosumab, or anabolic treatment is far more problematic and requires some in person appointments. Patients starting daily anabolic treatment need to have the injection method taught and demonstrated. The first injection of denosumab should be done in a clinic or hospital environment. After the first dose, it may be easier to have a caregiver inject it at home.

\section{Maintaining osteoporosis pharmacotherapy during the extended pandemic}

Maintenance of injected treatment during the pandemic is more challenging that of oral bisphosphonates. 


\section{Intravenous bisphosphonates}

The typical dosing of IV Zoledronic acid is annual. However, studies have shown that Zoledronate has a sustained duration of action; bone turnover markers remain suppressed beyond 1 year [29, 30]. Furthermore, randomized controlled trials indicate that less frequent dosing of zoledronic acid can still provide skeletal benefit and protection against fractures [27-29]. Hence, delays of even several months are unlikely to be harmful [29]. It will still be important to monitor and continue fall risk reduction techniques. In a few cases, it may be necessary to temporarily transition patients to oral bisphosphonates.

\section{Denosumab}

Patients on denosumab are the most problematic because of the known rebound vertebral fractures observed in patients who delay or miss doses [31]. Therefore, all major societies advocate that doses should not be delayed or missed [29]. Due to the continued pandemic, Amgen was offering short term home nurse injections in the U.S. Other creative solutions have included drive-in injection clinics, or using less crowded parts of hospital or clinics for injections. In some cases, denosumab has been shipped overnight to patients so that a family member could administer it.

If none of these solutions are possible, transitioning to an oral bisphosphonate within 7 months of the last denosumab injection should be considered [29]. It is general practice to measure serum calcium, creatinine, and/or 25-hydroxyvitamin D, before administration. The risk of hypocalcemia with denosumab is higher in patients with renal insufficiency. Without baseline renal insufficiency, this risk is low [29]. There has been concern about continuing denosumab in the face of COVID-19, because it is an immune system modulator, but a brief report did not find increased risk of COVID-19 in patients on denosumab [32].

\section{Romosozumab, teriparatide and abaloparatide}

Patients on teriparatide or abaloparatide have been educated on self-administration at home. However, Romosozumab is a once a month injection given in most clinic and hospital settings. There have been no reports of rebound fractures with interruption of romosozumab treatment, but patients clearly lose bone upon discontinuation of the anabolic agent. Most experts would suggest bisphosphonate options if there is disruption of anabolic treatment [29]. Anabolic agents need to be followed by anti-resorptives to maintain the increased bone; oral bisphosphonates will be the easiest anti-resorptive to start.

\section{Osteoporosis management principles as the pandemic continues}

COVID-19 will continue and maybe worsen until there is relief from vaccines. Prior to the pandemic there were substantial treatment gaps and projected increased fracture incidence because of aging populations, misapprehension of prescriptions' secondary side effects and belief that alternative or 'natural' treatments would suffice, leading to declining use of osteoporosis pharmacotherapy [33]. We need to be encourage colleagues in primary care and geriatrics to continue screening for osteoporosis as well as address fall risk in patients. We provide some potential ideas and methods to consider in osteoporosis care and management during the prolonged pandemic (Fig. 1).

\section{Creating triage systems: pinpoint the high risk}

\section{(A) Prioritizing bone densitometry screening}

A priority system for DXA is one sensible approach. Sapkota et al. suggest that low risk patients, based on FRAX questionnaires could have bone densitometry delayed. [34] Conversely, patients whose FRAX scores are $>20 \%$ for risk of major osteoporotic fractures or $>3 \%$ for hip fractures could proceed with empiric treatment without baseline bone densitometry testing. Individuals on androgen deprivation therapy, aromatase inhibitors, or chronic glucocorticoids have strong reasons for bone loss. Such populations should be risk stratified. The patient who has suffered an osteoporotic fracture does not need a DXA to make the diagnosis, an important message that needs to be reiterated. The DXA can be done later to help in determining the response to treatment. In addition, using a study cohort of 7959 women $\geq$ age 67 and age-adjusted proportional hazards models, Black et al. analyzed the relationships between a single baseline assessment of Femoral neck BMD, fracture history and age and 20-25 year fracture incidence. They concluded that a single BMD measurement and fracture history assessment may predict risk of fracture for up to 20-25 years [35]. Therefore, treatments may rely on previous DXA measurements and patients should not be left without treatment in absence of DXA follow-up.

\section{(B) Selecting face to face visits required for bone health care delivery}

Healthcare systems should determine which patients necessitate a face to face consultation versus which patients can have a telemedicine or phone visit. For osteoporosis, shortterm use of oral bisphosphonates provided patients have no 
Fig. 1 Ongoing osteoporosis management principles and efforts to consider and incorporate as we re-enter worldwide restrictions to local practices. $B M D$ bone mineral density; Fracture (Fx)

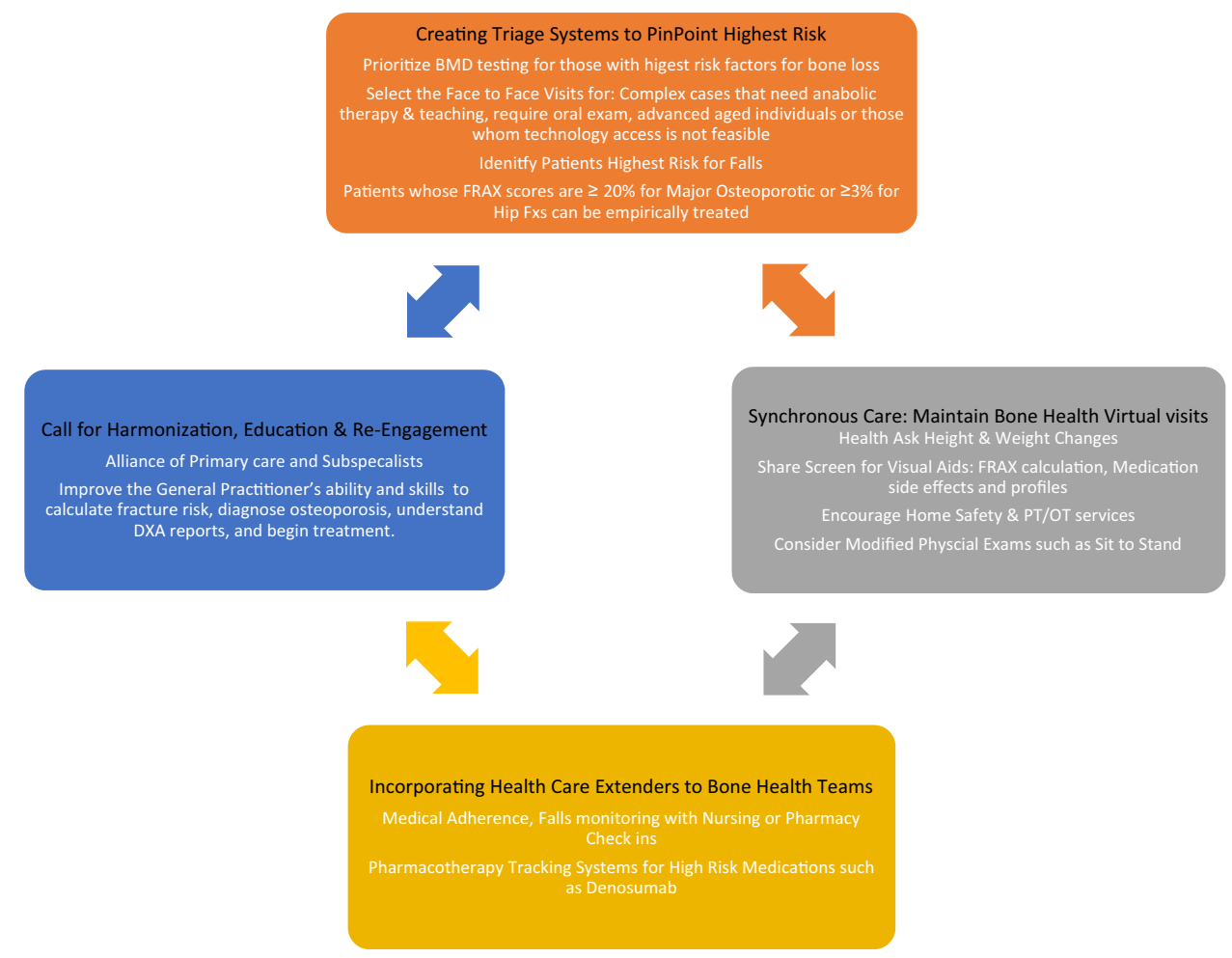

contraindications is prudent. However, if the original consultation is over the phone or telemedicine, there can be recognized challenges with an oral exam. If there is a question of poor dental hygiene or acute tooth or gum problems, such patients may require a face-to-face visit or a consultation to the dentist. Many dental practices provide safe assessments. Also, patients that require teaching for the daily injections of teriparatide or abaloparatide may need to physically come to clinics if virtual instruction with a nurse or pharmacist is not available. People with hearing, visual, or cognitive impairments and individuals who are resistant to or cannot afford technology may require in person visits.

\section{(C) Identify individuals with risk for falls and promote physical activity}

Falls can easily happen at home, especially when individuals are isolated from care givers or other support systems. Falls questionnaires such as STEADI may identify patients that need further interventions including physical and occupational therapies [36, 37]. Even on the phone, it is possible to ask a few but vital history questions about recent falls, activities of daily living (ADLs), or fear of falling. In addition, suggesting and promoting simple indoor activities to help reduce fall risk may be helpful. Examples are chair stands, going up and down stairs, dance, tai chi and yoga exercises.

\section{(D) Consider empiric treatment for high risk populations}

During the COVID-19 pandemic, patients with a history of fragility fracture can start treatment, even without formal BMD testing [33, 38]. Choosing the right agent may be particularly difficult at this time with limited laboratory assessments and testing capabilities but consider ease of use, availability of various drugs and patients' preferences. A recent meta-analysis of 20 trials found that bisphosphonates increase bone density and lower the occurrence of osteoporotic vertebral fractures in postmenopausal women without prior fracture history [39]. Because of extensive experience and general safety and efficacy, oral bisphosphonates can be considered as that initial bridge to reduce fracture risk in the prolonged COVID-19 era [33].

\section{Synchronous care: maximization of bone health virtual visits}

Telemedicine allows providers to 'see' the emotions of their patient and level of engagement, especially when describing new pharmacotherapy or changes to the osteoporosis treatment plans. Virtual visits add a meaningful advantage to telephone calls, especially for new patients. If telemedicine is not possible, a telephone call still permits continuation of 
care; thus patients do not feel abandoned. For osteoporosis, we can continue shared decision making approaches.

In addition, telemedicine offers selected patients more accessibility, especially for rural communities. Sub-Specialty Osteoporosis Centers are limited, making it necessary for some patients to travel long distances to reach expert physicians, even before COVID-19. The "old old" and/or those with disabilities likely have multiple comorbidities and complex osteoporosis management requirements. With the rapid implementation of telemedicine, we have witnessed elimination of long travel times and ease of access to expert care including osteoporosis specialists. Of course, some patients will not be able to manage the technical aspects of telemedicine.

For our patients requiring physical (PT) or occupational therapy (OT), telemedicine also allows therapists to see into people's homes, assess their environments, and instruct patients in home safety practices and home exercises. Physical therapy by telemedicine has been well received [40]. It is yet to be seen if patients will feel more "distant" in the virtual environment without tactile feedback of their exercise routines from therapists.

\section{(A) Tips for clinicians for osteoporosis telemedicine visits}

Telemedicine allows assessment of some risk factors for fracture and falls. FRAX questions can easily be ascertained, and most telemedicine platforms allow one to "share their screen" providing a visual FRAX assessment for the patient and family members. Typically, use of visual image aids boosts people's involvement in their treatment plans. For some patients, showing them a visual tool to contrast the risk of fracture with that of side effects, such as osteonecrosis of the jaw, will lessen patient concerns.

It is important to mimic the traditional face to face model as much as possible. Consider describing the physical exam out loud to the patient to keep them engaged in their care. Height and weight changes are essential vital signs. While the virtual physical exam clearly has limitations, it can be advantageous. Ask the patient to rise from sitting position to a standing position. If possible have the patient walk across the room, positioning their camera so you can assess gait. Certain clinics have already reviewed this as part of a modernized musculoskeletal physical examination and find it promising [41, 42]. Performing a portion of the physical exam conveys to the patient the extra effort to arrange a telemedicine visit. Some telemedicine is done in the patient's home; these may be called "virtual visits." More formal telemedicine may also take place in a facility near the patient but distant from the consulting clinician. Usually, there is a nurse or other medical person present who may be able to provide further physical assessment, while the consultant looks on from afar. This allows another excellent source of detailed information and does not constrain the patient or the clinician.

Lastly and most importantly, telemedicine requires redefining traditional patient-doctor interactions and relationships. We must adapt and modify our history taking, physical examinations, review of laboratory assessments and treatment plans with patients. Telemedicine allows us focused time to explain risks vs benefits of osteoporosis medications as part of comprehensive management.

\section{(B) Unknowns and remaining challenges with telemedicine and bone health}

While telemedicine is a vital tool, it is not known whether there is lasting benefit for long-term osteoporosis care. Will it affect adherence to osteoporosis medications? Elderly patients, who are more at risk for falls and fractures are less likely to be technically adept. For many, regular phone calls may be the only workable distant appointment method.

Fragmentation of care is an on-going problem for both in person and virtual medical visits. In a study from Canada prior to COVID-9, osteoporosis telemedicine visits were conducted for 5 years, and satisfaction questionnaires and telephone interviews were completed by the subjects. Patients reported satisfaction overall and noted it was similar in quality to face-to-face visits. Benefits mentioned included convenience and less travel. However, there was incoherent and disorganized follow up with referrals to other services such as physical therapy and with coordination of laboratory and imaging tests. The study excluded non-English speakers and people with decreased cognition [43]. The problems of fragmentation and lack of coordination occur with face to face visits as well, but perhaps the pandemic has highlighted these challenges. COVID-19 has made it harder for team member huddles and warm handoffs of care. The customary support of clinic personnel cannot be overstated. Redefining team-based care and multidisciplinary support with virtual care settings will be important and allow us to navigate and plan more efficient paths moving forward.

\section{Incorporating extenders into bone health teams}

Another possible intervention is utilizing healthcare extenders to improve medication adherence and screening for falls in between clinicians' visits. While the benefits of a team approach in fracture liaison services have been established, there is less information on whether a nurse (RN) or pharmacist phone clinic could improve osteoporosis medication compliance and adherence. In a UK rheumatology-led osteoporosis clinic, an $\mathrm{RN}$ telephone visit was offered rather than an in-person follow-up visit [44]. Seventy-one patients had an RN phone visit (average duration $10 \mathrm{~min}$ ). A majority 
(65\%) found it very convenient, $82 \%$ felt they had enough time to discuss their concerns and ask question, and $72 \%$ were happy and would use the service again. Wait times for follow-up in the rheumatology clinic were reduced by 2 months after implementation of phone visits. However, other studies did not show consistently improved adherence $[45,46]$. The stay home guidance during the COVID-19 pandemic may have an impact on the efficacy of phone visits. At a time where there is significant health care team member stress, finding ways to redistribute responsibilities and shared workloads may be more important than ever. No matter how effective a drug is, without the proper adherence to the recommended dosing regimen, the patient may not fully benefit from the course of therapy. Utilizing different patient adherence programs and team members to maintain a high level of patient compliance is necessary, critical and should be explored.

In addition, pharmacy dashboards have been integrated into electronic records for various chronic disease conditions and high risk medications $[47,48]$.We have not found published reports of such dashboards to monitor patients on osteoporosis medications such as denosumab or romosozumab. While physicians may express alert fatigue and ignore reminders, having a committed team member assigned to track these patients, remind them of appointments, and encourage medication adherence would improve resource utilization. The prolonged pandemic provides an opportunity to find cost-effective means to improve medication compliance and adherence.

\section{Call for Harmonization: outreach and Education in the extended COVID-19 pandemic}

Prior to the pandemic, it was obvious that most patients at risk for osteoporotic fracture would require management by primary care. The limited number of osteoporosis experts, competing medical problems, and now the prolonged pandemic have only increased the burden on primary care.

Suggestions for osteoporosis care during the pandemic are dependent on the many "stakeholders" of medical care. Insurance companies or government payment programs, patient preferences, information and disinformation from the internet, and fears of osteoporosis drugs compound the management of osteoporosis even in normal times. Primary care clinicians underestimate the impact of osteoporosis on quantity and quality of life. In this most challenging time, we must improve the general practitioner's ability to calculate fracture risk, diagnose osteoporosis, understand DXA reports, and begin treatment. The primary care clinician should be comfortable in prescribing oral bisphosphonates, be able to discuss side effects clearly and to utilize referrals to PT and OT. This may require new approaches and connections but is warranted as it enhances the reach of bone health care beyond the specialist capacity. For the patient already diagnosed and treated for osteoporosis, methods to continue treatment and re-assessment need to be instituted. While traditional continuing medical education (CME) programs have not led to adequate improvements in osteoporosis care, new ways and approaches to reduce fractures must be found. Using some of the proposed ideas in this paper as initial framework may help. We have to break the inertia and find ways to strengthen partnerships among health care delivery systems, public health professionals, osteoporosis sub-specialists and primary care colleagues.

One additional method to improve sub-specialty care is the electronic consultation (e-consult). The primary care provider can ask a question. The expert clinician completes a comprehensive review of the patient using the electronic record and provides recommendations. The specialist can offer an assessment and suggest treatment initiation with side effects outlined and monitoring guidelines. It is also an opportunity to actively teach clinicians key concepts in hopes they will learn and use this information for future patients. Real-time feedback can be a valuable and informative educational approach. E-consultations may also save time. Sub-specialists can recognize and triage the more complicated cases for Osteoporosis clinics to take over management. This has been shown to be useful in a study from Northern Ireland [49] and could be an opportunity for more institutions. Sharing of complicated patients, referral back to primary care when patient is stabilized, and use of mutually accessible records can improve osteoporosis care and distribute the care burden.

Finally, the impact of the extended pandemic on training of Osteoporosis Specialists is unknown. Gone are the days when medical students, residents, fellows and attending physicians would roam the wards or clinics together. The inability to have large teams working together in the medical settings could potentially hamper acquisition of certain clinical skills. Most institutional conferences have switched to virtual platforms. Programs such as bone health ECHOs and programs of many organizations such as the American Society for Bone and Mineral Research have turned to virtual modalities to showcase basic advancements, clinical research and case-based discussions. [50]. Optimization of precious clinician, educator, and trainee time to teaching is more difficult with the prolonged and serious pandemic. It will be challenging to produce and maintain a passionate and highly skilled workforce with metabolic bone expertise training. 


\section{Conclusions}

Global progress from the COVID-19 pandemic will come from preventive measures such as wearing masks and widespread vaccination efforts. There are growing concerns of mutant strains and the possibility of a more chronic epidemic. It is imperative to continue to recognize and treat osteoporosis and set long term strategies. Fracture patients, especially those with hip fracture, often undergo rehabilitation in long-term facilities. Such institutions have been major foci of COVID-19 infections. Preventing fracture lowers the population needing this long-term care. Prior to the pandemic, there was already less attention being paid to osteoporosis [51]. It is also difficult to predict what further inequities COVID-19 will amplify, including access to medical care for rural populations, individuals without internet or technology abilities, certain racial disparities and the challenges of an aging population. It will be challenging to re-engage the older population and clinicians with the importance of diagnosing osteoporosis. For patients already being treated, it will be necessary to reinforce the importance of continuing treatment until it is possible to determine whether treatment can be interrupted. Until enough people are vaccinated to lower infection rates dramatically, attention to other important disorders, such as osteoporosis, will remain difficult.

It is our duty as osteoporosis advocates to do what we can to alleviate patient concerns, strive to remain proactive and committed to working with and across teams for prevention of fractures and the long-term disease burden of osteoporosis.

\section{Compliance with ethical standards}

Conflict of interest None.

\section{References}

1. Sözen T, Özışık L, Başaran N (2017) An overview and management of osteoporosis. Eur J Rheumatol 4(1):46-56

2. Borgström F, Karlsson L, Ortsäter G, Norton N, Halbout P, Cooper C, Lorentzon M, McCloskey EV, Harvey NC, Javaid MK, Kanis JA (2020) Fragility fractures in Europe: burden, management and opportunities. Arch Osteoporos 15(1):59

3. Clynes MA, Harvey NC, Curtis EM, Fuggle NR, Dennison EM, Cooper C (2020) The epidemiology of osteoporosis. Br Med Bull 133(1):105-117

4. Messina C, Buzzoni AC, Gitto S, Almolla J, Albano D, Sconfienza LM (2020) Disruption of bone densitometry practice in a Northern Italy Orthopedic Hospital during the COVID-19 pandemic. Osteoporos Int 5:1-5

5. Kanis JA, Harvey NC, Cooper C, Johansson H, Odén A, McCloskey EV (2016) A systematic review of intervention thresholds based on FRAX: a report prepared for the National Osteoporosis Guideline Group and the International Osteoporosis Foundation. Arch Osteoporos 11(1):25

6. McCloskey EV, Harvey NC, Johansson H, Lorentzon M, Vandenput L, Liu E, Kanis JA (2020) Global impact of COVID-19 on non-communicable disease management: descriptive analysis of access to FRAX fracture risk online tool for prevention of osteoporotic fractures. Osteoporos Int 6:1-8

7. Ogliari G, Lunt E, Ong T, Marshall L, Sahota O (2020) The impact of lockdown during the COVID-19 pandemic on osteoporotic fragility fractures: an observational study. Arch Osteoporos 15(1):156

8. Gumina S, Proietti R, Polizzotti G, Carbone S, Candela V (2020) The impact of COVID-19 on shoulder and elbow trauma: an Italian survey. J Shoulder Elbow Surg 29(9):1737-1742

9. Arafa M, Nesar S, Abu-Jabeh H, Jayme MOR, Kalairajah Y (2020) COVID-19 pandemic and hip fractures: impact and lessons learned. Bone Jt Open 1(9):530-540

10. Malik-Tabassum K, Crooks M, Robertson A, To C, Maling L, Selmon G (2020) Management of hip fractures during the COVID-19 pandemic at a high-volume hip fracture unit in the United Kingdom. J Orthop 20:332-337

11. Zhang B, Zhang S (2020) Corticosteroid-Induced Osteonecrosis in COVID-19: a call for caution. J Bone Miner Res 35(9):1828-1829

12. di Filippo L, Formenti AM, Doga M, Pedone E, Rovere-Querini P, Giustina A (2020) Radiological thoracic vertebral fractures are highly prevalent in COVID-19 and predict disease outcomes. J ClinEndocrinolMetab 6:1-13

13. Dy CJ, Lane JM, Pan TJ, Parks ML, Lyman S (2016) Racial and socioeconomic disparities in hip fracture care. J Bone Joint Surg Am 98(10):858-865

14. Wright NC, Chen L, Saag KG, Brown CJ, Shikany JM, Curtis JR (2020) Racial disparities exist in outcomes after major fragility fractures. J Am GeriatrSoc 68(8):1803-1810

15. Cauley JA (2011) Defining ethnic and racial differences in osteoporosis and fragility fractures. ClinOrthopRelat Res 469(7):1891-1899

16. Cram P, Saag KG, Lou Y, Edmonds SW, Hall SF, Roblin DW, Wright NC, Jones MP, Wolinsky FD (2017) Racial differences and disparities in osteoporosis-related bone health: results from the paadrn randomized controlled trial. Med Care 55(6):561-568

17. Bilezikian JP, Brandi ML, Eastell R, Silverberg SJ, Udelsman R, Marcocci C, Potts JT Jr (2014) Guidelines for the management of asymptomatic primary hyperparathyroidism: summary statement from the fourth international workshop. J ClinEndocrinolMetab 99(10):3561-3569

18. Lombardi CP, D'Amore A, Grani G, Ramundo V, Boscherini M, Gordini L, Marzi F, Tedesco S, Bocale R (2020) Endocrine surgery during COVID-19 pandemic: do we need an update of indications in Italy? Endocrine 68(3):485-488

19. Jozaghi Y, Zafereo ME, Perrier ND, Wang JR, Grubbs E, Gross ND, Fisher S, Sturgis EM, Goepfert RP, Lai SY, Best C, Busaidy NL, Cabanillas ME, Dadu R, Gagel RF, Habra MA, Hu MI, Jimenez C, Sherman SI, Thosani S, Varghese J, Waguespack SG, Weitzman S, Ying AK, Graham PH (2020) Endocrine surgery in the Coronavirus disease 2019 pandemic: surgical triage guidelines. Head Neck 42(6): 1325-1328

20. Abrishami A, Dalili N, MohammadiTorbati P, Asgari R, ArabAhmadi M, Behnam B, Sanei-Taheri M (2020) Possible association of vitamin D status with lung involvement and outcome in patients with COVID-19: a retrospective study. Eur J Nutr 4:1-9

21. Hernández JL, Nan D, Fernandez-Ayala M, García-Unzueta M, Hernández-Hernández MA, López-Hoyos M, Muñoz-Cacho P, Olmos JM, Gutiérrez-Cuadra M, Ruiz-Cubillán JJ, Crespo J, Martínez-Taboada VM (2020) Vitamin D status in hospitalized 
patients with SARS-CoV-2 infection. J ClinEndocrinolMetab $1: 172-134$

22. Maghbooli Z, Sahraian MA, Ebrahimi M, Pazoki M, Kafan S, Tabriz HM, Hadadi A, Montazeri M, Nasiri M, Shirvani A, Holick MF (2020) Vitamin D sufficiency, a serum 25-hydroxyvitamin D at least $30 \mathrm{ng} / \mathrm{mL}$ reduced risk for adverse clinical outcomes in patients with COVID-19 infection. PLoS ONE 15(9):e0239799

23. Bilezikian JP, Bikle D, Hewison M, Lazaretti-Castro M, Formenti AM, Gupta A, Madhavan MV, Nair N, Babalyan V, Hutchings N, Napoli N, Accili D, Binkley N, Landry DW, Giustina A (2020) Mechanisms in endocrinology: vitamin D and COVID-19. Eur J Endocrinol 183(5):R133-r147

24. Waldron JL, Ashby HL, Cornes MP, Bechervaise J, Razavi C, Thomas OL, Chugh S, Deshpande S, Ford C, Gama R (2013) Vitamin D: a negative acute phase reactant. J ClinPathol 66(7):620-622

25. Tramontana F, Napoli N, El-Hajj Fuleihan G, Strollo R (2020) The D-side of COVID-19: musculoskeletal benefits of vitamin D and beyond. Endocrine 69(2):237-240

26. Jin JM, Bai P, He W, Wu F, Liu XF, Han DM, Liu S, Yang JK (2020) Gender differences in patients with COVID-19: focus on severity and mortality. Front Public Health 8:152

27. Lundholm MD, Poku C, Emanuele N, Emanuele MA, Lopez N (2020) SARS-CoV-2 (COVID-19) and the endocrine system. J Endocr Soc 4(11):bvaa144

28. Smetana K Jr, Rosel D, Br Ábek J (2020) Raloxifene and bazedoxifene could be promising candidates for preventing the COVID-19 related cytokine storm. ARDS Mortality In Vivo 34(5):3027-3028

29. Yu EW, Tsourdi E, Clarke BL, Bauer DC, Drake MT (2020) Osteoporosis management in the era of COVID-19. J Bone Miner Res 35(6):1009-1013

30. Reid IR, Horne AM, Mihov B, Stewart A, Garratt E, Wong S, Wiessing KR, Bolland MJ, Bastin S, Gamble GD (2018) Fracture prevention with zoledronate in older women with osteopenia. $\mathrm{N}$ Engl J Med 379(25):2407-2416

31. Anastasilakis AD, Polyzos SA, Makras P, Aubry-Rozier B, Kaouri S, Lamy O (2017) Clinical features of 24 patients with rebound-associated vertebral fractures after denosumab discontinuation: systematic review and additional cases. J Bone Miner Res 32(6):1291-1296

32. Formenti AM, Pedone E, di Filippo L, Ulivieri FM, Giustina A (2020) Are women with osteoporosis treated with denosumab at risk of severe COVID-19? Endocrine 70(2):203-205

33. Napoli N, Elderkin AL, Kiel DP, Khosla S (2020) Managing fragility fractures during the COVID-19 pandemic. Nat Rev Endocrinol 16(9):467-468

34. Sapkota HR, Nune A, Bateman J, Venkatachalam S (2020) A pragmatic proposal for triaging DXA testing during the COVID19 global pandemic. Osteoporos Int 4:1-6

35. Black DM, Cauley JA, Wagman R, Ensrud K, Fink HA, Hillier TA, Lui LY, Cummings SR, Schousboe JT, Napoli N (2018) The ability of a single BMD and fracture history assessment to predict fracture over 25 years in postmenopausal women: the study of osteoporotic fractures. J Bone Miner Res 33(3):389-395

36. Stevens JA, Phelan EA (2013) Development of STEADI: a fall prevention resource for health care providers. Health PromotPract 14(5):706-714

37. Phelan EA, Mahoney JE, Voit JC, Stevens JA (2015) Assessment and management of fall risk in primary care settings. Med Clin North Am 99(2):281-293

38. Conley RB, Adib G, Adler RA, Åkesson KE, Alexander IM, Amenta KC, Blank RD, Brox WT, Carmody EE, ChapmanNovakofski K, Clarke BL, Cody KM, Cooper C, Crandall CJ, Dirschl DR, Eagen TJ, Elderkin AL, Fujita M, Greenspan SL, Halbout P, Hochberg MC, Javaid M, Jeray KJ, Kearns AE, King T, Koinis TF, Koontz JS, Kužma M, Lindsey C, Lorentzon M, Lyritis
GP, Michaud LB, Miciano A, Morin SN, Mujahid N, Napoli N, Olenginski TP, Puzas JE, Rizou S, Rosen CJ, Saag K, Thompson E, Tosi LL, Tracer H, Khosla S, Kiel DP (2020) Secondary fracture prevention: consensus clinical recommendations from a multistakeholder coalition. J Bone Miner Res 35(1):36-52

39. Wu CH, Hung WC, Chang IL, Tsai TT, Chang YF, McCloskey EV, Watts NB, McClung MR, Huang CF, Chen CH, Wu KL, Tsai KS, Chan DC, Chen JF, Tu ST, Hwang JS, Xia W, Matsumoto T, Chung YS, Cooper C, Kanis JA, Yang RS, Chan WP (2020) Pharmacologic intervention for prevention of fractures in osteopenic and osteoporotic postmenopausal women: systemic review and meta-analysis. Bone Rep 13:100729

40. Tenforde AS, Borgstrom H, Polich G, Steere H, Davis IS, Cotton K, O'Donnell M, Silver JK (2020) Outpatient physical, occupational, and speech therapy synchronous telemedicine: a survey study of patient satisfaction with virtual visits during the COVID19 pandemic. Am J Phys Med Rehabil 99(11):977-981

41. Tanaka MJ, Oh LS, Martin SD, Berkson EM (2020) Telemedicine in the era of COVID-19: the virtual orthopaedic examination. $J$ Bone Joint Surg Am 102(12):e57

42. Laskowski ER, Johnson SE, Shelerud RA, Lee JA, Rabatin AE, Driscoll SW, Moore BJ, Wainberg MC, Terzic CM (2020) The telemedicine musculoskeletal examination. Mayo ClinProc 95(8):1715-1731

43. Palcu P, Munce S, Jaglal SB, Allin S, Chishtie JA, Silverstein A, Kim S (2020) Understanding patient experiences and challenges to osteoporosis care delivered virtually by telemedicine: a mixed methods study. Osteoporos Int 31(2):351-361

44 Hennell S, Spark E, Wood B, George E (2005) An evaluation of nurse-led rheumatology telephone clinics. Musculoskeletal Care 3(4):233-240

45. Solomon DH, Iversen MD, Avorn J, Gleeson T, Brookhart MA, Patrick AR, Rekedal L, Shrank WH, Lii J, Losina E, Katz JN (2012) Osteoporosis telephonic intervention to improve medication regimen adherence: a large, pragmatic, randomized controlled trial. Arch Intern Med 172(6):477-483

46. van den Berg P, van Haard PMM, van der Veer E, Geusens PP, van den Bergh JP, Schweitzer DH (2018) A dedicated Fracture Liaison Service telephone program and use of bone turnover markers for evaluating 1-year persistence with oral bisphosphonates. Osteoporos Int 29(4):813-824

47. Peterson JF, Kripalani S, Danciu I, Harrell D, Marvanova M, Mixon AS, Rodriguez C, Powers JS (2014) Electronic surveillance and pharmacist intervention for vulnerable older inpatients on high-risk medication regimens. J Am GeriatrSoc 62(11):2148-2152

48. Waitman LR, Phillips IE, McCoy AB, Danciu I, Halpenny RM, Nelsen CL, Johnson DC, Starmer JM, Peterson JF (2011) Adopting real-time surveillance dashboards as a component of an enterprisewide medication safety strategy. Jt Comm J Qual Patient Saf 37(7):326-332

49 Lindsay JR, Lawrenson G, English S (2020) A service evaluation of e-triage in the osteoporosis outpatient clinic-an effective tool to improve patient access? Arch Osteoporos 15(1):53

50. Lewiecki EM, Rothman MS (2020) COVID-19, medical education, and bone health: insights from project ECHO. J ClinDensitom 23(3):338-339

51. Lewiecki EM, Wright NC, Curtis JR, Siris E, Gagel RF, Saag KG, Singer AJ, Steven PM, Adler RA (2018) Hip fracture trends in the United States, 2002 to 2015. Osteoporos Int 29(3):717-722

Publisher's Note Springer Nature remains neutral with regard to jurisdictional claims in published maps and institutional affiliations. 